\title{
T1 high-grade bladder carcinoma outcome: the role of p16, topoisomerase-II $\alpha$, survivin, and E-cadherin ${ }^{\text {th }}$
}

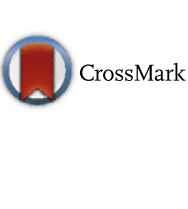

\section{Maria Rosaria Raspollini MD, PhD ${ }^{a, *}$, Rafael J. Luque MD ${ }^{b}$, Carmen Luz Menendez MD ${ }^{c}$, Enrico Bollito MD ${ }^{d}$, Matteo Brunelli MD ${ }^{e}$, Guido Martignoni MD ${ }^{e}$, Rodolfo Montironi MD, FRCPath, IFCAP ${ }^{f}$, Liang Cheng MD ${ }^{g}$, Ana Blanca MD ${ }^{h}$, Gianna Baroni BSc ${ }^{a}$, Andrea Minervini MD, PhD ${ }^{i}$, Antonio Lopez-Beltran MD, PhD ${ }^{j, k}$}

\author{
${ }^{a}$ Histopathology and Molecular Diagnostics, University Hospital Careggi, Florence, Italy \\ ${ }^{\mathrm{b}}$ UGC Anatomía Patológica, Complejo Hospitalario de Jaén, Jaén, Spain \\ ${ }^{\mathrm{c}}$ Departamento de Anatomia Patologica, Hospital de Cabueñes, Gijon, Asturias, Spain \\ ${ }^{\mathrm{d}}$ Division of Pathology University of Turin, San Luigi Gonzaga Hospital, Orbassano, Turin, Italy \\ 'Anatomic Pathology, Department of Pathology, University of Verona, Verona, Italy \\ Region, School of Medicine, United Hospitals, Ancona, Italy \\ Cordoba, Spain \\ i Department of Urology, University Hospital Careggi, Florence, Italy \\ ${ }^{\mathrm{j} U n i t}$ of Anatomical Pathology, Department of Surgery, Faculty of Medicine, Cordoba, Spain \\ ${ }^{\mathrm{k}}$ Champalimaud Clinical Center, Lisbon, Portugal
}

${ }^{\mathrm{f} S e c t i o n}$ of Pathological Anatomy, Department of Biomedical Sciences and Public Health Polytechnic University of the Marche

${ }^{\mathrm{g}}$ Department of Pathology and Laboratory Medicine, Indiana University School of Medicine, Indianapolis, IN, 46202, USA

${ }^{\mathrm{h}}$ Urology Department, Maimonides, Institute of Biomedical Research (IMIBIC), Reina Sofia Hospital, University of Cordoba,

Received 5 April 2016; revised 5 June 2016; accepted 28 June 2016

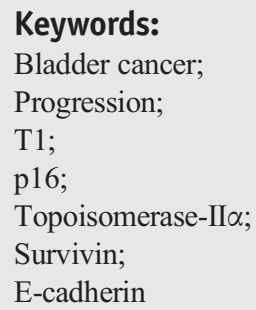

\begin{abstract}
Summary High-grade papillary urothelial carcinoma with subepithelial connective tissue invasion (T1HG) is an aggressive disease at high risk of progression after transurethral resection/Bacillus Calmette-Guerin standardized therapy. The European Organization for Research and Treatment of Cancer has identified T1HG bladder carcinoma that is single and $\leq 3 \mathrm{~cm}$ in the largest dimension at first diagnosis as a category in which the prognosis cannot be further stratified based on conventional criteria. This category may benefit from biomarker analysis as a valuable tool to determine the patient's outcome. To further the issue of biomarkers in predicting aggressiveness in single T1HG bladder carcinoma $\leq 3 \mathrm{~cm}$ in greatest dimension at first diagnosis, we have conducted a validation study of the biomarker risk score set previously reported by our group. The study set included immunohistochemical detection of galectin-3, CD44, E-cadherin (ECAD), CD138, p16, survivin, HYAL-1, and topoisomerase-II $\alpha$ in 92 randomly selected specimens at participating institutions. Topoisomerase-II $\alpha$ expression was identified as a predictor of disease-free survival. p16, survivin, and E-CAD expression predicted progression-free survival, but p16 and E-CAD also predicted
\end{abstract}

\footnotetext{
is Disclosures: The authors declared no potential conflicts of interest with respect to and received no financial support for the research, authorship, and/or publication of this article.

* Corresponding author at: Histopathology and Molecular Diagnostics, University Hospital Careggi, Largo Brambilla, 3-50134, Florence, Italy.

E-mail address: mariarosaria.raspollini@unifi.it (M. R. Raspollini).
} 
overall survival. The current study validates a panel of immunohistochemical markers with the potential of being implemented in practice and supports the use of biomarkers in predicting aggressiveness in patients with first diagnosis of single T1HG bladder carcinoma $\leq 3 \mathrm{~cm}$ in greatest dimension and therefore in identifying patients who need closer surveillance or earlier aggressive treatment.

(C) 2016 Elsevier Inc. All rights reserved.

\section{Introduction}

High-grade (HG) papillary urothelial carcinoma with subepithelial connective tissue invasion (T1 disease) represents about $20 \%$ of bladder urothelial carcinomas. After standardized therapy, up to $75 \%$ of patients with $\mathrm{T} 1$ disease will experience tumor recurrence within 2 years. Importantly, up to $25 \%$ of these tumors have a high risk of progression to invasive disease (T2-4) [1]. To identify the most aggressive subset of cases would be of great relevance in clinical practice $[2,3]$.

The European Organization for Research and Treatment of Cancer (EORTC) risk tables may predict $\mathrm{pTa}$ and $\mathrm{pT} 1$ bladder tumor recurrence and progression including different variables such as tumor diameter, T stage, time to recurrence, association with urothelial carcinoma in situ (CIS), and grade. Furthermore, urothelial pT1HG bladder carcinoma less than $3 \mathrm{~cm}$ (greatest diameter) at first diagnosis is not amenable to stratification into prognostic categories because its behavior is not predictable [4]. No prognostic molecular markers, at this stage, have been included in clinical management [5]. The utilization of biomarkers can accurately predict progression in patients with non-muscle-invasive tumors and help identify patients who need closer surveillance or aggressive treatment. This might be very useful in the clinic [6-8].

Studies have shown independent prediction of recurrence and progression of non-muscle-invasive bladder carcinoma by Ki-67, p53, pRb, p21, and p27 $[9,10]$.

We previously reported a biomarker-based risk score based on the immunohistochemical evaluation of galectin-3, CD44, E-cadherin (E-CAD), CD138, p16, survivin, HYAL-1, and topoisomerase-II $\alpha$ (TOPO- $2 \alpha$ ) to predict tumor progression in patients with $<3-\mathrm{cm}$ single tumors [11]. The current study aimed to validate our biomarker-based risk score in a large series of T1 high-grade cases from different institutions and to assess their potential application in practice.

\section{Materials and methods}

\subsection{Patients}

The study series included 92 cases of T1 high-grade bladder cancer collected from participating institutions with the following histological and clinical inclusion criteria:

(1) histologic diagnosis of HG papillary urothelial carcinoma T1 with tumor size $\leq 3 \mathrm{~cm}$ and with presence of muscularis propria at the first transurethral resection of the bladder (TUR-
B); (2) previous negative history of urothelial tumor; (3) repetitive transurethral resection of the bladder post-Bacillus Calmette-Guerin (BCG) therapy showing no residual disease; and (4) known follow-up.

We collected the materials from 6 departments of pathology of different European hospitals; all slides were reassessed by 2 specialized pathologists (A. L. B. and M. R. R.) to confirm the diagnosis of the tumor grade, the presence of muscularis propria, the presence of CIS, T1 substaging, and the presence or absence of tumor necrosis. Subsequently, appropriate immunohistochemical analysis was performed on selected representative samples.

Recurrence was defined as the finding of new non-muscleinvasive lesions after a complete resection and induction course with BCG, whereas progression was defined as the recurrence of a muscle-invasive lesion after TUR-B and BCG instillations.

\subsection{Tissue specimens and immunohistochemistry}

The specimens were fixed in $10 \%$ formalin before being processed in paraffin. Hematoxylin-eosin-stained sections from each histologic specimen were reviewed to confirm the histologic diagnosis of T1HG urothelial carcinoma or CIS. The slides and tissue blocks from first TUR-Bs were used for the immunohistochemical analysis.

The following immunohistochemical markers were evaluated: galectin-3, CD44, E-CAD, CD138, p16, survivin, HYAL-1, and TOPO-2 $\alpha$. The primary antibodies anti-galectin-3 (clone 9C4, ready to use), anti-E-CAD (clone EP700Y, ready to use), anti-CD138/syndecan-1 (clone B-A38, ready to use), and anti-p16INK4a CINtec histology (clone E6H4, ready to use, Ventana, Tucson, AZ) were placed on the slides and incubated on the Ventana automated stainer BenchMark ULTRA ICH system. For chromogenic detection, ultraView Universal DAB Detection Kit (Ventana Medical Systems, Tucson, AZ) was used. Additional serial sections were immunostained with rabbit monoclonal anti-survivin (clone EP2880Y, ready to use), anti-TOPO- $2 \alpha$ rabbit monoclonal (cloneEP1102Y, ready to use) anti-CD44 (H-CAM, clone DF1485, ready to use, Biogenex, Fremont, CA), and antiHYAL-1 rabbit polyclonal (dilution 1:100, Novus Biologicals, Littleton, CO). Antigen retrieval was carried out with Epitope Retrieval Solution Citrate buffer $(10 \mathrm{mmol} / \mathrm{L}, \mathrm{pH}$ 6; Dako, Glostrup, Denmark) in thermostatic bath (PT Link, Pre-Treatment Module; Dako). Anti-TOPO- $2 \alpha$ and antisurvivin were incubated for 1 hour at room temperature, antiCD44 for 2 hours at room temperature, and anti-HYAL-1 overnight at $4{ }^{\circ} \mathrm{C}$. The immunohistochemical analyses were 


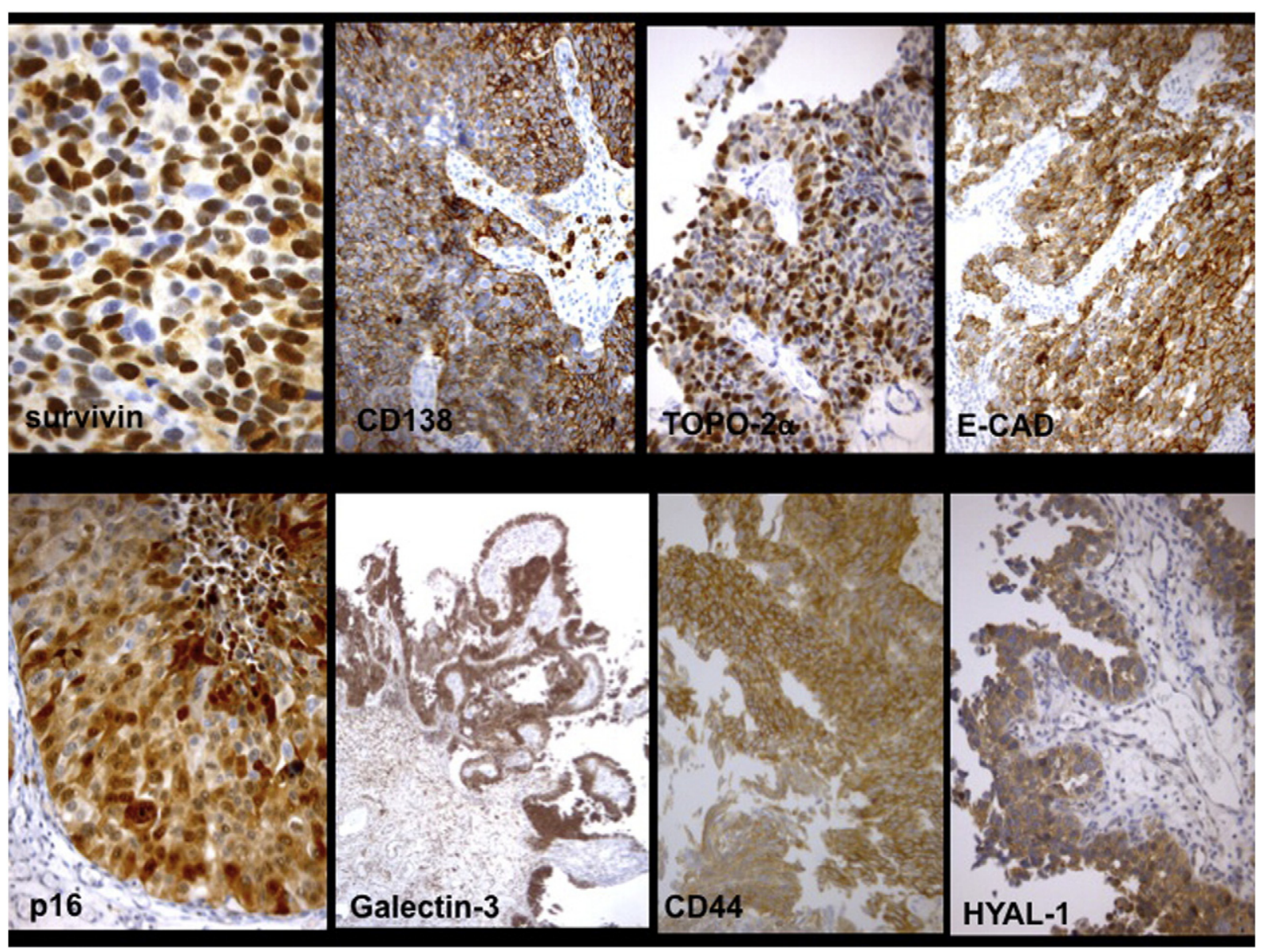

Fig. 1 Examples of immunohistochemically determined biomarkers in the current study. Anti-galectin-3 (original magnification $\times 20$ ), antiCD44 $(\times 10)$, anti-E-CAD $(\times 10)$, anti-CD138 $(\times 10)$, anti-p16 $(\times 20)$, anti-survivin $(\times 10)$, anti-HYAL-1 $(\times 10)$, and anti-TOPO-2 $\alpha(\times 10)$.

performed using EnVision FLEX Systems (Dako) and 3,3'diaminobenzidine (Dako) as chromogen in Dako Autostainer Link48 Instrument.

Sections of strongly positive tonsil (CD138), papillary carcinoma of thyroid (galectin-3), lung adenocarcinoma (E-CAD), colorectal adenoma (CD44), urinary bladder carcinoma (survivin), endometrial glands (HYAL-1), and uterine cervix carcinoma (TOPO-2 $\alpha$ ) were used as positive controls. Negative control was performed with FLEX Negative Control Mouse and FLEX Negative Control Rabbit (ready to use, Dako). The control sections were treated in parallel with the samples. The sections were lightly counterstained with Mayer hematoxylin.

\subsection{Biomarker expression and evaluation of immunostaining}

The stains for HYAL-1 and galectin-3 were considered positive if any cytoplasmic staining was seen in the neoplastic tissue. A cytoplasm staining with a membranous-reinforced staining pattern was required for considering CD138 positivity. A membrane staining was required for E-CAD and CD44. The stains for survivin and TOPO- $2 \alpha$ were considered positive if nuclear staining was seen in the neoplastic tissue. For survivin, we evaluated only the nuclear-labeling index because previous studies demonstrated the nuclear positivity of this marker to be a prognostic tool for $\mathrm{Ta} / \mathrm{T} 1$ urothelial carcinoma of the bladder [12].

The stain for $\mathrm{p} 16$ was considered positive if nucleus and cytoplasm or only nucleus staining was seen in the tumor cells. For galectin-3, CD44, and E-CAD, we used the von Brunn nests as positive internal control, whereas for the other markers, we used the von Brunn nests as negative internal control. Fig. 1 shows positive staining of the studied markers.

All slides were evaluated by 2 uropathologists (A. L. B. and M. R. R.) in a blind evaluation. For semiquantitative assessment of the immunohistochemical data, the mean percentage of positive tumor cells was determined at $\times 400$ magnification for each section. Sections were graded on the basis of the percentage of tumor cells. The following were considered negative: no tumor cells with positive nuclei for survivin, TOPO- $2 \alpha$, and p16; no tumor cells with cytoplasm staining with a membranous-reinforced staining pattern for CD138; no tumor cells with cytoplasmatic staining for HYAL-1 and galectin-3, or no tumor cells with membrane staining for E-CAD and CD44. 
Table 1 Demographic and pathologic characteristics of patients with T1 high-grade bladder cancer included in the current study

\begin{tabular}{|c|c|}
\hline Characteristics & $\mathrm{n}(\%)$ \\
\hline Age (y), median (range) & $72.2(70.2-74.2)$ \\
\hline \multicolumn{2}{|l|}{ Sex } \\
\hline Female & $12(13.0 \%)$ \\
\hline Male & $80(87.0 \%)$ \\
\hline \multicolumn{2}{|l|}{$\mathrm{T}$ category substaging } \\
\hline $\mathrm{T} 1$ focal & $42(45.7)$ \\
\hline T1 extensive & $50(54.3)$ \\
\hline \multicolumn{2}{|l|}{ Concomitant CIS } \\
\hline No & $66(71.7)$ \\
\hline Yes & $26(28.3)$ \\
\hline \multicolumn{2}{|l|}{ Focal tumor necrosis } \\
\hline No & $77(83.7)$ \\
\hline Yes & $15(16.3)$ \\
\hline \multicolumn{2}{|l|}{ Tumor recurrence } \\
\hline No & $65(70.7)$ \\
\hline Yes & $27(29.3)$ \\
\hline \multicolumn{2}{|l|}{ Tumor progression } \\
\hline No & $75(81.5)$ \\
\hline Yes & $17(18.5)$ \\
\hline \multicolumn{2}{|l|}{ Overall survival } \\
\hline Alive & $65(70.7)$ \\
\hline Dead of bladder cancer & $14(15.2)$ \\
\hline Dead, other cause & $13(14.1)$ \\
\hline p16, median (range) & $39.4(30.5-48.2)$ \\
\hline CD138, median (range) & $85.16 \pm 17.62$ \\
\hline TOPO-2 $\alpha$, median (range) & $36.7(31.9-41.4)$ \\
\hline Survivin, median (range) & $37(32-42)$ \\
\hline Galectin-3, median (range) & $56(50-62.5)$ \\
\hline CD44, median (range) & $13.8(7.9-19.6)$ \\
\hline HYAL-1, median (range) & $31.6(24.9-38.4)$ \\
\hline E-CAD, median (range) & $78.7(73.4-84.1)$ \\
\hline
\end{tabular}

\subsection{Statistical analysis}

Statistical analysis was performed using SPSS (Chicago, IL) 21.0 software at the Jaén Hospital Research Unit, Spain (R. J. L.). A descriptive analysis was performed, and then receiver operating curve analyses were performed for each marker to find reliable cutoff values, selecting those with a greater sensitivity and specificity for recurrence, progression, and disease-related mortality. After determination of cutoff points, association between variables was tested with Fisher exact test. Univariate survival analysis was conducted using the Kaplan-Meier method, with differences among groups being tested for significance using the log-rank test. Multivariate analysis of probable prognostic factors for survival was performed with Cox proportional hazard regression analysis, and the relative risk with $95 \%$ confidence interval was calculated. A $P$ less than .05 was considered statistically significant.

\section{Results}

We evaluated the specimens from transurethral resection of 92 patients with T1HG bladder carcinoma that was single and $\leq 3 \mathrm{~cm}$ in greatest dimension at first diagnosis. Main demographic and clinicopathological data of the patients as well as the mean expression of biomarkers in the study are summarized in Table 1. Most patients in the study were male (87\%), and $28 \%$ of patients had concomitant CIS. On follow-up ranging $13-170$ months, $29 \%, 18.5 \%$, and $15 \%$ of patients recurred, progressed, or died of disease, respectively.

Table 2 presents univariate survival analysis of the patients in this series. The current study identified TOPO- $2 \alpha$ expression (cutoff at 30\%) as predictor of disease-free survival $(P=.021)$ (Fig. 2). p16 (cutoff at 60\%) $(P=.04$ ), survivin (cutoff at $50 \%)(P=.012)$, and E-CAD (cutoff at 90\%) $(P=.007)$ immunoexpressions predicted progression-free survival (Fig. 3). In addition, p16 (cutoff at 60\%) $(P=.009)$ and survivin (cutoff at $50 \%)(P=.000)$ predicted overall survival (Fig. 4).

In the multivariate analysis (Table 3 ), TOPO- $2 \alpha$ was selected as an independent predictor of disease-free survival $(P=.029)$, and survivin was selected as an independent predictor of progression-free survival $(P=.020)$. However, survivin $(P=$ $.006)$ and $\mathrm{E}-\mathrm{CAD}(P=.030)$ were selected as independent predictors of overall survival in the current study.

None of the other investigated biomarkers (galectin-3, CD44, CD138, HYAL-1) was found to be correlated to survival outcome in the current study.

\section{Discussion}

In this study, we confirmed that some biomarkers can support risk assessment in high-grade bladder cancer with early stromal invasion. In fact, there is a subset of these tumors in which predictors of aggressiveness are very limited as demonstrated by the EORTC studies. Currently, conventional predictors cannot predict aggressiveness in primarily diagnosed single T1HG urothelial carcinoma less than or equal to $3 \mathrm{~cm}$ in diameter; likewise, available data show that these patients have $35 \%$ probability of recurrence and $10 \%$ probability of progression at 24 months of initial diagnosis. Therefore, our group has suggested that using biomarker expression could potentially provide additional information of value in predicting aggressiveness in this particular group of tumors.

In the current biomarker study, we have seen that a panel including TOPO- $2 \alpha$ expression, p16, survivin, and E-CAD might predict survival in single pT1HG less than or equal to $3 \mathrm{~cm}$ in diameter. Immunohistochemical expression of $\mathrm{p} 16$, survivin, and E-CAD at given cutoff points correlated with progression-free survival, and it correlated with increasingly aggressive outcome and increased risk of tumor progression. Similarly, immunohistochemical expression of $\mathrm{p} 16$ and survivin turned out to be predictors of overall survival in the univariate analysis performed in the current study. Interestingly, survivin 
Table 2 Univariate survival analysis according to Kaplan-Meier method and the log-rank test

\begin{tabular}{|c|c|c|c|c|c|c|c|c|c|c|}
\hline & Overall & $\begin{array}{l}\text { Disease-free } \\
\text { survival }\end{array}$ & $\begin{array}{l}\text { Log- } \\
\text { rank }\end{array}$ & $P$ & $\begin{array}{l}\text { Progression-free } \\
\text { survival }\end{array}$ & $\begin{array}{l}\text { Log- } \\
\text { rank }\end{array}$ & $P$ & $\begin{array}{l}\text { Overall } \\
\text { survival }\end{array}$ & $\begin{array}{l}\text { Log- } \\
\text { rank }\end{array}$ & $P$ \\
\hline p16 & & & 2.022 & .155 & & 3.773 & .049 & & 6.917 & .009 \\
\hline$<60 \%$ & 56 & 20 & & & 7 & & & 4 & & \\
\hline$>60 \%$ & 36 & 7 & & & 10 & & & 10 & & \\
\hline CD138 & & & 0.155 & .694 & & 0.985 & .321 & & 0.545 & .460 \\
\hline$<70 \%$ & 13 & 3 & & & 1 & & & 3 & & \\
\hline$>70 \%$ & 79 & 24 & & & 16 & & & 11 & & \\
\hline Topoisomerase & & & 5.302 & .021 & & 0.093 & .761 & & & \\
\hline$<30 \%$ & 30 & 13 & & & 6 & & & 6 & 1.436 & .231 \\
\hline$>30 \%$ & 62 & 14 & & & 11 & & & 8 & & \\
\hline Survivin & & & 0.233 & .629 & & 6.337 & .012 & & 16.632 & .000 \\
\hline$<50 \%$ & 65 & 19 & & & 8 & & & 4 & & \\
\hline$>50 \%$ & 27 & 8 & & & 9 & & & 10 & & \\
\hline Galectin & & & 1.089 & .297 & & 0.334 & .563 & & 0.128 & .721 \\
\hline$<50 \%$ & 37 & 14 & & & 8 & & & 7 & & \\
\hline$>50 \%$ & 55 & 13 & & & 9 & & & 7 & & \\
\hline CD44 & & & 0.445 & .505 & & 1.775 & .183 & & 0.746 & .388 \\
\hline Negative & 65 & 18 & & & 10 & & & 9 & & \\
\hline Positive & 27 & 9 & & & 7 & & & 5 & & \\
\hline Hyaluronidase & & & 0.345 & .557 & & 0.072 & .788 & & 0.278 & .598 \\
\hline$<70 \%$ & 72 & 22 & & & 13 & & & 10 & & \\
\hline$>70 \%$ & 20 & 5 & & & 4 & & & 4 & & \\
\hline E-CAD & & & 2.266 & .132 & & 7.270 & .007 & & 2.645 & .104 \\
\hline$<90 \%$ & 46 & 16 & & & 3 & & & 3 & & \\
\hline$>90 \%$ & 46 & 11 & & & 14 & & & 11 & & \\
\hline
\end{tabular}

remained as an independent predictor of overall survival, together with E-CAD, which became independent with a minor decreasing of expression, as low as $10 \%$ reduction. We also observed that the immunohistochemical expression of TOPO$2 \alpha$ when seen in more than $30 \%$ of cancer cells is a positive predictor of disease-free survival.

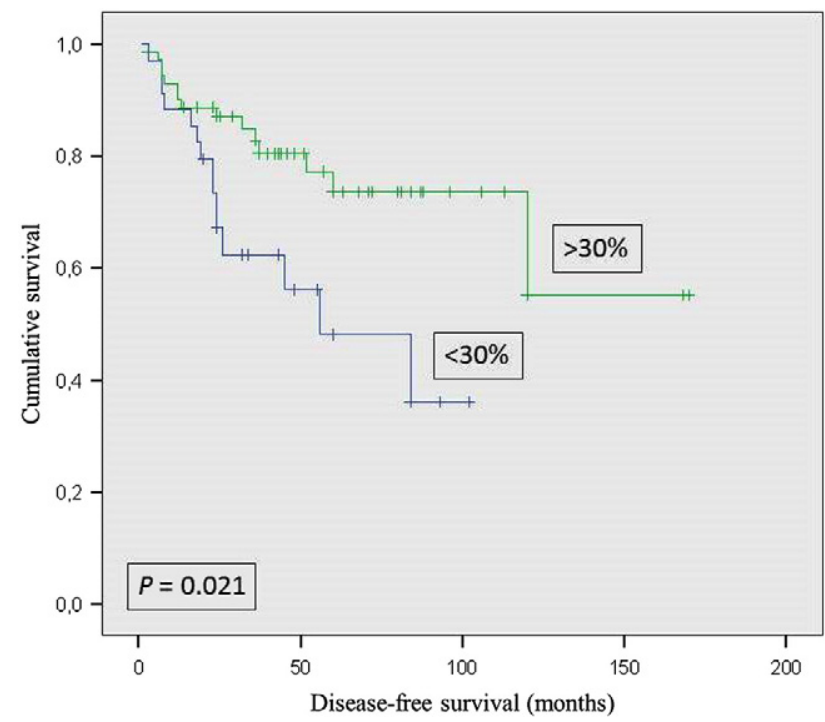

Fig. 2 Kaplan-Meier plots of disease-free survival according to immunoexpression of TOPO- $2 \alpha$.
These results are not surprising because p16 is a protein encoded by $C D K N 2 A$ oncosuppressor gene, which belongs to the CDKI family; its function is to restrict the activity of kinases. This protein is able to stop the cellular cycle and to restrict mitotic activity to assist in the process of DNA repair [13]. The gene encoding p16 is mutated or downregulated in several cancer cells. Breast carcinoma progression has been related to overexpression of p16 [14], and this has been reported in head and neck squamous carcinoma [15], in prostate carcinoma [16], and also in aggressive subtypes of bladder carcinoma [17-20].

Survivin is a member of the inhibitors of the apoptosis family. The survivin protein limits caspase activation, leading to a negative regulation of apoptosis [21]. Cases of highly aggressive pT1 bladder carcinoma can be identified by high survivin expression [22-24].

TOPO- $2 \alpha$, a DNA gyrase isoform, plays an important role in the cell cycle and catalyzes the isomerization of DNA by facilitating the passage of 1 strand of DNA through a reversible break in the second strand of DNA. Deregulation or qualitative alterations of the TOP- $2 \alpha$ expression during the cell cycle have been reported in both normal tissue and various human neoplasms [25]. Enhanced expression of TOP-2 $\alpha$ is positively associated with a high rate of recurrence and progression in primary non-muscle-invasive bladder cancer [26].

Finally, among the genetic alterations, the different expression of the cadherin family seems to have a link with bladder cancer [27]. The cadherin family includes N-, P-, and E-CAD. Only 

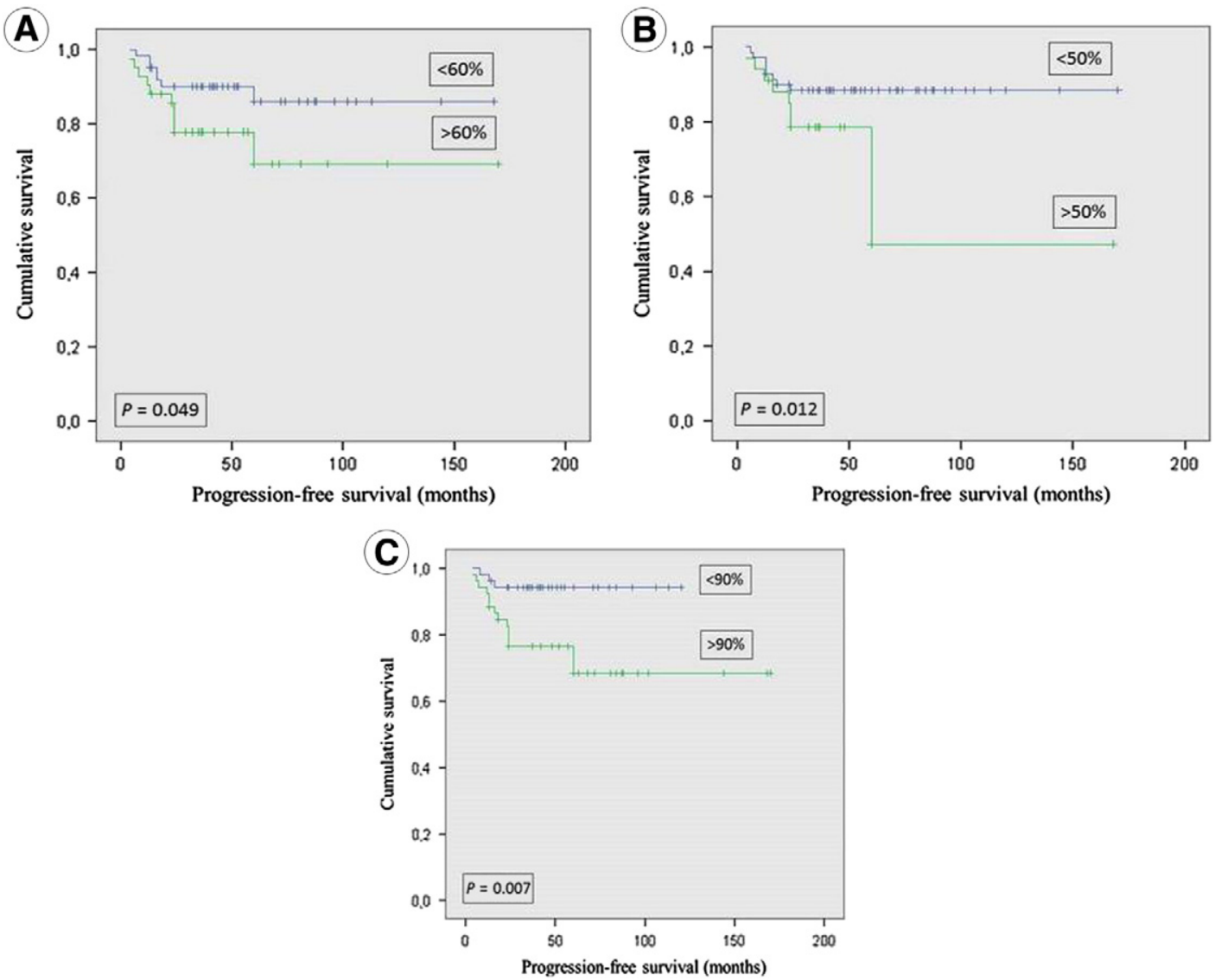

Fig. 3 Kaplan-Meier plots of progression-free survival according to immunoexpression of p16 (A), survivin (B), and E-CAD (C).

E-CAD, a 120-kDa glycoprotein, the gene of which is located on chromosome 16, is associated with intracellular proteins called catenins that mediate between extracellular signals and cytoskeleton microfilaments. The loss of E-CAD expression is associated with a loss of cellular differentiation and increased cellular invasiveness [28,29], and it may correlate with muscle involvement and tumor recurrence. Loss of E-CAD is also described in some aggressive subtypes of urothelial carcinoma of the bladder [30].

In conclusion, our study shows that we were able to identify a series of easily applied immunohistochemical biomarkers to offer further predictive information in addition to conventional prognostic parameters used in the EORTC scoring system; and this may be useful in identifying patients at high risk of
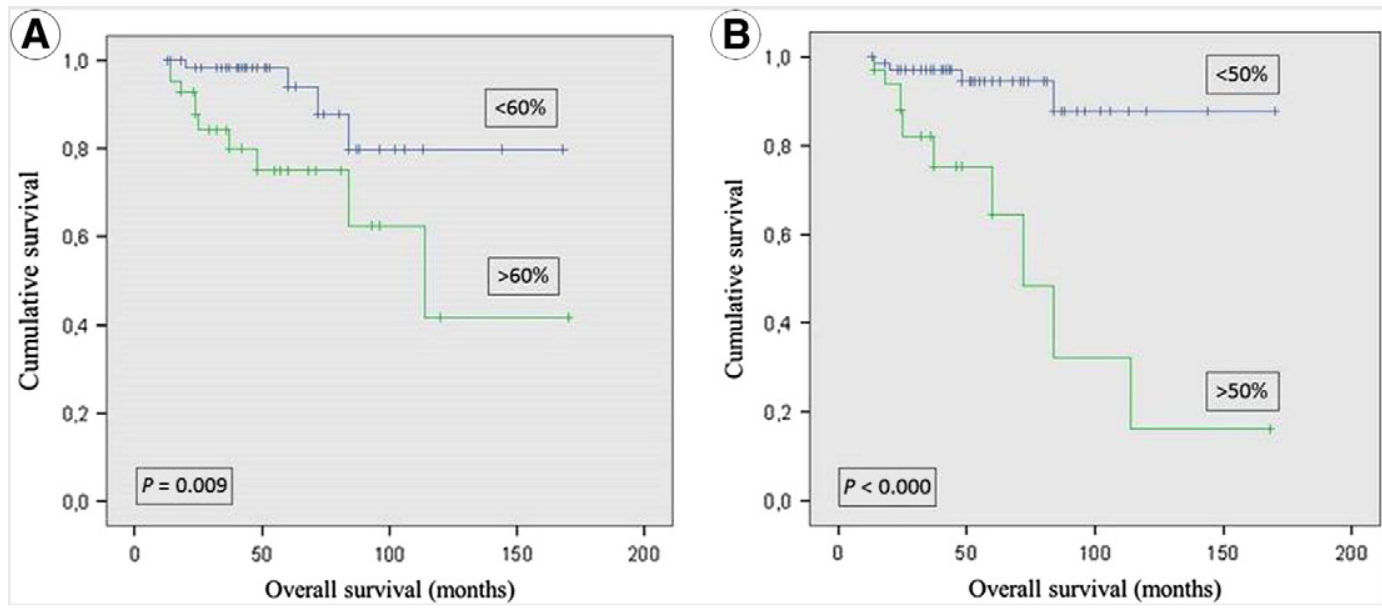

Fig. 4 Kaplan-Meier plots of overall survival according to immunoexpression of p16 (A) and survivin (B). 
Table 3 Cox multivariate statistical analysis showing independent variables related to disease-free, progression-free, and overall survival of patients with $\mathrm{T} 1$ high-grade bladder cancer

\begin{tabular}{llr}
\hline & $P$ & \multicolumn{1}{c}{ RR } \\
\hline Disease-free survival & & \\
$\quad$ TOPO- $\alpha \leq 30 \%$ $\alpha s>30 \%$ & .029 & .978 \\
Progression-free survival & & \\
$\quad$ Survivin $\leq 50 \%$ vs $>50 \%$ & .020 & 1.024 \\
Overall survival & & \\
$\quad$ Survivin $\leq 50 \%$ v $>50 \%$ & .006 & 6.175 \\
$\quad$ E-CAD $\leq 90 \%$ v $>90 \%$ & .030 & 10.958 \\
\hline Abbreviation: RR, relative risk. & &
\end{tabular}

progression or of death from disease. This particular set of biomarkers includes TOPO- $2 \alpha$, p16, survivin, and E-CAD, and if properly investigated may assist to accurately predict survival probability in single $\mathrm{T} 1$ high-grade urothelial carcinoma less than $3 \mathrm{~cm}$ in diameter.

\section{References}

[1] Kurth KH, Denis L, Bouffioux C, et al. Factors affecting recurrence and progression in superficial bladder tumors. Eur J Cancer 1995;31A: 1840-6.

[2] van Rhijn BW, Burger M, Lotan Y, et al. Recurrence and progression of disease in non-muscle-invasive bladder cancer: from epidemiology to treatment strategy. Eur Urol 2009;56:430-42.

[3] Liu W, Qi L, Zu X, et al. A preoperative marker panel for the prediction of residual tumor and the decision making for repeat transurethral resection. Urol Oncol 2015;33:165.e9-165.e14.

[4] Sylvester RJ, Van der Meijden AP, Oosterlinck W, et al. Predicting recurrence and progression in individual patients with stage TaT1 bladder cancer using EORTC risk tables: a combined analysis of 2596 patients from seven EORTC trials. Eur Urol 2006;49:466-77.

[5] Babjuk M, Oosterlinck W, Sylvester R, et al. EAU guidelines on nonmuscle-invasive urothelial carcinoma of the bladder, the 2011 update. Eur Urol 2011;59:997-1008.

[6] van Rhijn BW, Musquera M, Liu L, et al. Molecular and clinical support for a four-tiered grading system for bladder cancer based on the WHO 1973 and 2004 classifications. Mod Pathol 2015;28:695-705.

[7] van Rhijn BW. Combining molecular and pathologic data to prognosticate non-muscle-invasive bladder cancer. Urol Oncol 2012;30:518-23.

[8] Kim PH, Cha EK, Sfakianos JP, et al. Genomic predictors of survival in patients with high-grade urothelial carcinoma of the bladder. Eur Urol 2015;67:198-201.

[9] Lopez-Beltran A, Luque RJ, Alvarez-Kindelan J, et al. Prognostic factors in stage T1 grade 3 bladder cancer survival: the role of G1-S modulators (p53, p21Waf1, p27kip1, cyclin D1, and cyclin D3) and proliferation index (ki67-MIB1). Eur Urol 2004;45:606-12.

[10] Quintero A, Alvarez-Kindelan J, Luque RJ, et al. Ki-67 MIB1 labelling index and the prognosis of primary TaT1 urothelial cell carcinoma of the bladder. J Clin Pathol 2006;59:83-8.

[11] Raspollini MR, Minervini A, Lapini A, et al. A proposed score for assessing progression in $\mathrm{pT} 1$ high-grade urothelial carcinoma of the bladder. Appl Immunohistochem Mol Morphol 2013;21:218-27.
[12] Yin W, Chen N, Zhang Y, et al. Survivin nuclear labeling index: a superior biomarker in superficial urothelial carcinoma of human urinary bladder. Mod Pathol 2006;19:1487-97.

[13] Kotake Y, Naemura M, Murasaki C, Inoue Y, Okamoto H. Transcriptional regulation of the p16 tumor suppressor gene. Anticancer Res 2015;35: 4397-401.

[14] Pare R, Shin JS, Lee SC. Increased expression of senescence markers p14(ARF) and p16 (INK) (4a) in breast cancer is associated with increased risk of disease recurrence and poor survival outcome. Histopathology 2016;69:479-91.

[15] Baruah P, Lee M, Wilson PO, et al. Impact of p16 status on pro- and antiangiogenesis factors in head and neck cancers. Br J Cancer 2015;113: 653-9.

[16] Zhang Z, Rosen DG, Yao JL, Huang J, Liu J. Expression of p14ARF, p15INK4b, p16INK4a, and DCR2 increases during prostate cancer progression. Mod Pathol 2006;19:1339-43.

[17] Yin M, Bastacky S, Parwani AV, et al. p16ink4 immunoreactivity is a reliable marker for urothelial carcinoma in situ. Нuм РАтноL 2007;39: 527-35.

[18] Buza N, Cohen PJ, Hui P, et al. Inverse p16 and p63 expression in small cell carcinoma and high-grade urothelial cell carcinoma of the urinary bladder. Int J Surg Pathol 2010;18:94-102.

[19] Abat D, Demirhan O, Inandiklioglu N, et al. Genetic alterations of chromosomes, $\mathrm{p} 53$ and p16 genes in low- and high-grade bladder cancer. Oncol Lett 2014;8:25-32.

[20] Kim SH, Joung JY, Chung J, Park WS, Lee KH, Seo HK Detection of human papillomavirus infection and p16 immunohistochemistry expression in bladder cancer with squamous differentiation. PLoS One 2014;9:e93525. http://dx.doi.org/10.1371/journal.pone. 0093525 [eCollection 2014].

[21] Ambrosini G, Adida C, Altieri DC. A novel anti-apoptosis gene, survivin, expressed in cancer and lymphoma. Nat Med 1997;3: 917-21.

[22] Weiss C, Von Romer F, Capalbo G, et al. Survivin expression as a predictive marker for local control in patients with high-risk T1 bladder cancer treated with transurethral resection and radio-chemotherapy. Int J Radiat Oncol Biol Phys 2009;74:1455-60.

[23] Kim K, Sung CO, Park BH, et al. Immunoprofile-based subgrouping of urothelial bladder carcinomas for survival prediction. Нuм РАтноL 2015; 46:1464-70.

[24] Kim K, Cho YM, Park BH, et al. Histological and immunohistochemical markers for progression prediction in transurethrally resected high-grade non-muscle invasive bladder cancer. Int J Clin Exp Pathol 2015;8: $743-50$

[25] Schaefer-Klein JL, Murphy SJ, Johnson SH, Vasmatzis G, Kovtun IV. Topoisomerase 2 alpha cooperates with androgen receptor to contribute to prostate cancer progression. PLoS One 2015;10:e0142327.

[26] Ben Abdelkrim S, Rammeh S, Ziadi S, Tlili T, Jaidane M, Mokni M. Expression of topoisomerase II alpha, ki67, and p53 in primary nonmuscle-invasive urothelial bladder carcinoma. J Immunoassay Immunochem 2014;35:358-67.

[27] Breyer J, Gierth M, Shalekenov S, et al. Epithelial-mesenchymal transformation markers E-cadherin and survivin predict progression of stage pTa urothelial bladder carcinoma. World J Urol 2016;34:709-16.

[28] Canas-Marques R, Schnitt SJ. E-cadherin immunohistochemistry in breast pathology: uses and pitfalls. Histopathology 2016;68:57-69.

[29] Dabbs DJ, Schnitt SJ, Geyer FC, et al. Lobular neoplasia of the breast revisited with emphasis on the role of E-cadherin immunohistochemistry. Am J Surg Pathol 2013;37:e1-11

[30] Raspollini MR, Sardi I, Giunti L, et al. Plasmacytoid urothelial carcinoma of the urinary bladder: clinicopathologic, immunohistochemical, ultrastructural, and molecular analysis of a case series. Нuм РАтноц 2011:42:1149-58. 